\title{
Educación Intercultural Bilingüe en La Araucanía: principales limitaciones epistemológicas ${ }^{1}$
}

\author{
Katerin Arias-Ortega ${ }^{2}$ \\ ORCID: 0000-0001-8099-0670 \\ Daniel Quilaqueo ${ }^{2}$ \\ ORCID: 0000-0002-5143-4920 \\ Segundo Quintrique0² \\ ORCID: 0000-0002-7228-4095
}

\section{Resumen}

El tema central del artículo es una revisión del estado de arte de la educación intercultural, sustentado en las siguientes interrogantes: ¿La educación intercultural bilingüe responde a las demandas del pueblo mapuche en relación a una educación con pertinencia sociocultural?, ¿Considera otras reivindicaciones de tipo política y económica que surgen desde el Estado chileno? El objetivo es develar los alcances y limitaciones epistemológicas de la educación intercultural en La Araucanía. La metodología consistió en una revisión de la literatura normativa y científica a nivel nacional e internacional, para lograr una comprensión y explicación del problema. El enfoque teórico es la interculturalidad crítica en educación. Se concluye que para avanzar en una educación intercultural desde una epistemología contextual es necesario: 1) generar espacios en la institución escolar para la co-construcción de materiales didácticos que sistematicen contenidos educativos propios, con el apoyo de profesores, familia y comunidad; 2) incorporar métodos educativos mapuches propios en la implementación de la educación intercultural; 3) favorecer el trabajo colaborativo con los miembros de la comunidad que tienen conocimientos sobre oficios, para articular el conocimiento teórico y práctico en la sala de clases, desde la mirada indígena-occidental; y 4) promover procesos de enseñanza-aprendizaje que se realicen a partir de actividades de indagación tanto en la comunidad como en la familia y en la escuela.

\section{Palabras clave}

Educación Intercultural - Desafíos epistémicos - Epistemología contextual.

1- Agradecimientos: Al Programme des futurs leaders dans les Amériques (PFLA) del Gobierno de Canadá. Al proyecto Fondecyt №1181314 y No1181531.

2- Universidad Católica de Temuco. Temuco, Chile.

Contactos: ariaskaterin@gmail.com; dquilaq@uct.cl; squintri@uct.cl.

(c) (i) Dol: http://dx.doi.org/10.1590/S1678-4634201945192645

This content is licensed under a Creative Commons attribution-type BY-NC. 


\section{Intercultural Bilingual Education in La Araucanía: main epistemological limitations}

\section{Abstract}

The core topic of the article is a review of the state of the art of intercultural education, based on the following questions: Does intercultural bilingual education respond to the demands of the Mapuche people in relation to a sociocuturally relevant education? Does it consider other political and economic demands that arise from the Chilean State? The objective is to reveal the scope and epistemological limitations of intercultural education in La Araucanía. The methodology consisted of a review of the normative and scientific literature at a national and international level, to achieve an understanding and explanation of the problem. The theoretical approach is critical interculturality in education. It is concluded that to advance intercultural education from a contextual epistemology it is necessary 1) to create spaces in the schools for the co-construction of teaching materials that systematize their own educational content, with the support of teachers, family, and community; 2) to incorporate Mapuche educational methods of their own in the implementation of intercultural education; 3) to promote collaborative work with community members who have knowledge of trades, in order to articulate the theoretical and practical learning in the classroom, from the western-indigenous perspective; y 4) to promote teaching-learning processes that are built on research activities both in the community, in the family, and at school.

\section{Keywords}

Intercultural education - Epistemic challenges - Contextual epistemology.

\section{Introducción}

Sostenemos que la Educación Intercultural Bilingüe (EIB), desarrollada en La Araucanía, presenta limitaciones epistemológicas en la definición de contenidos, métodos y finalidades educativas en su implementación. Por una parte, se observa la invisibilización de los aportes epistémicos de la educación mapuche en la educación escolar, para la formación de niños y jóvenes mapuches y no mapuches y, por otra parte, que los mapuches han ingresado a la educación escolar como medio de supervivencia y estrategia para luchar contra la exclusión y desigualdades sociales de la sociedad dominante (SERRANO; PONCE DE LEÓN; RENGIFO, 2012; SILVA-PEÑA et al., 2013). Sin embargo, el resultado del ingreso a la educación escolar también ha traído un desarraigo social, cultural y lingüístico, puesto que la mayoría de los padres de familia escolarizados han adoptado la cultura escolar como única y válida para la formación de sus hijos. Esto evidencia cómo la escuela chilena ha influido, ya que actualmente la mayoría de los padres de familia 
no transmite sus propios conocimientos. En consecuencia, se constata la desvalorización de la diversidad social y cultural indigena existentes en el país, particularmente entre los mapuches en La Araucanía. Observamos que esto es el resultado de relaciones interétnicas que las instituciones estatales y privadas han establecido con las personas e instituciones sociales mapuches. Dichas relaciones están marcadas por la negación y desprecio de los conocimientos educativos mapuches en la escuela, de manera explícita e implícita (QUILAQUEO; TORRES, 2013), aunque mediante la implementación de la EIB se ha impulsado el reconocimiento oficial de la lengua mapuche en la educación escolar.

Se observa, además, que la incorporación de algunos conocimientos educativos mapuches al aula, con la implementación de la EIB, se ha realizado en base a métodos educativos escolares tradicionales, es decir, la memorización y reproducción literal de contenidos, negando con ello procesos reflexivos que faciliten la comprensión y atribución de significados a los conocimientos educativos indígenas. Así, sostenemos, que estas prácticas educativas reproducen el formato escolar hegemónico de la escuela, naturalizando los espacios, sus tiempos, organización curricular, lenguaje visual y métodos educativos escolares, que se configuran en elementos que mantienen las relaciones de poder y dominación hacia los indígenas (CANDAU, 2016). Desde esta mirada, emergen tensiones y conflictos epistemológicos que no permiten al profesorado, en su quehacer pedagógico, establecer una relación más dialógica entre ambas lógicas de pensamientos y conocimientos, durante el desarrollo de la educación intercultural. Lo anterior, considerando que la escuela al estar inserta en comunidades mapuches, en las que existe una base epistémica de saberes y conocimientos educativos propios, se vería beneficiada si incorpora dichos conocimientos a la educación escolar, para fortalecer y potenciar el desarrollo de la EIB, desde una epistemología contextual. Pensamos que visibilizar la base de conocimientos mapuche que convergen en el contexto comunitario y escolar posibilitaría un diálogo de saberes. Así como su sistematización en tanto contenidos educativos, para revertir progresivamente los mecanismos de homogeneización de la escuela. Puesto que la escuela históricamente se ha atribuido el derecho de imponer una lógica de pensamiento monocultural de corte eurocéntrico occidental sobre los diferentes pueblos indígenas y grupos inmigrantes.

La lógica monocultural refiere a tres aspectos principales: 1) la práctica monolingüe del español, desarrollada en los procesos de enseñanza aprendizaje, que niega la incorporación de otra lengua, como el mapunzugun (lengua mapuche), al sistema escolar; 2) las prácticas que excluyen las minorías religiosas y étnicas en el desarrollo de los procesos educativos escolares; y 3) el uso del currículum de corte eurocéntrico, que propicia el desarrollo de los conocimientos y valores que la sociedad occidental considera válidos; invisibilizando los saberes, conocimientos y valores que poseen los estudiantes de diferentes sociedades y culturas (SARANGAPANI, 2014; MAMPAEY; ZANONI, 2015; QUINTRIQUE0 et al., 2017). Sostenemos que la lógica monocultural que se desprende de estas tres prácticas de monoculturalidad, en contexto de colonización, termina minorizando y tensionando la relación de los sujetos que se desenvuelven en contextos indígenas y de diversidad cultural. Consecuentemente, constituyen prácticas que limitan el desarrollo de una epistemología contextualizada a las realidades sociales y culturales de cada pueblo, para contextualizar la educación escolar en perspectiva intercultural. 
Lo eurocéntrico refiere a la racionalidad que impuso sólo una forma de producir conocimiento, colonizando y sobreponiéndose a saberes y conocimientos concretos de pueblos indigenas y latinoamericanos descendientes de colonos europeos (QUIJANO, 1992, 2014). Esto ha generado discriminación, racismo y prejuicios contra los indígenas (MANSILLA; BECERRA; MERINO, 2015), lo que continúa actualmente con prácticas de exclusión y minorización de estos pueblos (VAN DIJK, 2010; QUINTRIQUE0; TORRES, 2012; CORREA; SALDARIAGA, 2014; MAMPAEY; ZANONI, 2015). Esto perpetúa la jerarquización del conocimiento occidental como conocimiento superior y, junto con ello, establece una idea de progreso y orden moral que no considera las formas y métodos educativos propios de los pueblos indígenas para construir conocimientos (SALAÜN, 2013; QUILAQUEO; QUINTRIQUEO; PEÑA-CORTÉS, 2016). De este modo, se limitan las posibilidades de diálogo y se avanza en la fragmentación e instrumentalización de la epistemología indígena, despojándola de sus prácticas y significados, para fortalecer la colonización mental de los individuos en contextos indígenas. Así la colonización mental instala, entre las personas, la idea de que se puede construir conocimiento válido y científico sólo desde lo europeo (CORREA; SALDARIAGA, 2014).

Desde los argumentos planteados, surgen tres preguntas contrapuestas: ¿Qué buscan los pueblos indígenas con las demandas de educación intercultural para sus hijos?; ¿Es una mejor educación occidental o es una educación sustentada en sus propios conocimientos? y ¿Qué buscan los Estados-nación con la implementación de una educación intercultural bilingüe: reforzar la educación escolar o incorporar las epistemes indígenas al currículum escolar? Para dar respuesta a estas interrogantes, en relación a la propuesta de la EIB en La Araucanía, proponemos las teorías emergentes de interculturalidad crítica (FORNETBETANCOURT, 2001; OLIVÉ, 2009; SANTOS, 2009) y de educación intercultural (WALSH, 2012a; QUINTRIQUEO et al., 2015; TUBINO, 2014).

La metodología empleada en este trabajo consistió en un mapeo sistemático de artículos científicos en el contexto internacional, nacional y local que dan cuenta de resultados de investigación y experiencias educativas respecto de la educación intercultural y su implementación en el sistema educativo escolar en contexto indígena. Posteriormente, el corpus de artículos científicos se categorizó en tres dimensiones. En una primera instancia, se separó la literatura normativa asociada a leyes, normas y decretos que se constituyen en marcos regulatorios que sustenta la educación intercultural a nivel internacional y nacional; se revisaron diez normativas. En segunda instancia se separó la literatura científica referida a artículos y resultados de investigación en cuatro idiomas principalmente, inglés, francés, portugués y español, constituyendo un total de cien artículos revisados. Finalmente, la literatura correspondiente a divulgación científica como son los libros; se revisaron diez experiencias. En tanto se revisaron diez capítulos de libros, los cuales se constituyen en la última categorización y revisión establecida. Esto se justifica debido a la accesibilidad de la información. Es así como, de un total de 130 referencias revisadas se seleccionaron 67 de ellos sustentan el presente ensayo.

El procedimiento de análisis de la información consistió en el análisis de contenido, revelando los significados implícitos y explícitos que subyacen en el texto (SAYAG0, 2014), respecto de la educación intercultural y su implementación en contextos indígenas 
e interculturales. Desde la perspectiva de Quilaqueo, Quintriqueo y Peña Cortés (2016) el procedimiento de análisis de contenido permite la complementariedad metodológica. En este caso fue la teoría fundamentada, puesto que implica desarrollar de manera articulada una codificación abierta otorgando núcleos de sentido a los contenidos que subyacen aesta. Así, el objetivo del presente artículo es develar los alcances y limitaciones epistemológicas sobre el desarrollo de la educación intercultural bilingüe en La Araucanía.

\section{Educación Intercultural Bilingüe}

La EIB surge en América Latina en la década de los sesenta del siglo pasado, como respuesta educativa desde los estados nacionales a las demandas de los pueblos indígenas respecto de una educación con pertinencia social y cultural, que incorpore sus saberes y conocimientos educativos propios a la institución escolar (LÓPEZ, 2007; WALSH, 2012b; TUBINO, 2013). Sin embargo, la EIB en América Latina no abandona su estructura colonial, su propósito civilizatorio, ni la base epistémica eurocéntrica occidental, que sustenta el conocimiento educativo escolar impuesto a los pueblos indigenas (OSORIO; APARECIDA, 2016).

El desarrollo de la EIB en Chile y en la región de La Araucanía es similar al de América Latina: es presentada como modalidad educativa en tanto política pública dirigida solamente a la población indigena. Surge como respuesta a las demandas educativas históricas planteadas por organizaciones sociales mapuches, desde principio del siglo XIX hasta la década de los ochenta (LÓPEZ; KÜPER, 2000; LLANQUINA0, 2009; QUINTRIQUEO; MUÑOZ, 2015). En ese contexto, en el año 1993, mediante la promulgación de la Ley Indígena n. 19.253, se reconoce a los pueblos indígenas con sus especificidades lingüísticas y culturales propias, y obliga al Estado chileno a respetar y proteger a las culturas y lenguas indígenas (HEVIA; HIRMAS, 2005). Con esta ley se pretende revertir el trato discriminatorio que ha tenido el Estado chileno con el pueblo mapuche.

Así, la EIB se ha implementado desde un enfoque educativo escolar que se enfoca en la diferenciación social, aumentando las desigualdades sociales, la dominación, la exclusión y la relación asimétrica de poder entre la población indígena y no indígena (NASCIMENTO; QUADROS; FIALHO, 2016). Por ello se configura en un elemento político que busca la reproducción de estructuras de discriminación social, lo cual se devela en función del bajo impacto de la EIB en las escuelas que la implementan. Según Williamson (2012), en La Araucanía la implementación de la EIB reproduce la estructura social y educacional de discriminación y desigualdad, lo que se refleja en un impacto negativo en la mejora de ingresos económico y el acceso al empleo de la población mapuche, en comparación a los no mapuches. Esta realidad es similar a lo ocurrido en México, según Viveros Márquez y Moreno Olivos (2014), donde la incorporación de profesores indígenas para implementar la EIB no ha permitido mejorar la calidad educativa de los estudiantes, puesto que está enfocada en responder a las exigencias del sistema educativo nacional. Es por ello que la enseñanza de la lengua vernácula se realiza en español. Asimismo, se carece de recursos didácticos, lo cual incide en el aprendizaje de la lengua vernácula. En coherencia con lo anterior, en Chile, un estudio encargado por el Ministerio de Educación 
(CHILE, 2012), sostiene que a pesar de la implementación de la EIB el dominio de la lengua mapunzugun ha disminuido sistemáticamente en la población en general. Por ejemplo, en el grupo de niños en edad escolar de 0 a 17 años, entre los años 2009 al 2011, la pérdida progresiva del mapunzugun aumenta en un 50\%. Esto se debe a las relaciones de asimetría y tensión entre lo dispuesto por la política pública y las condiciones en que se ha desarrollado la educación intercultural bilingüe (RIEDEMANN, 2008; TURRA, FERRADA; VILLENA, 2013).

El análisis de esta realidad nos permite sostener que la escuela no ha cambiado su finalidad de avanzar en la colonización y civilización de los indígenas, sustentado en una educación monocultural eurocéntrica que transmite la hegemonía del conocimiento escolar (QUINTRIQUEO et al., 2015). Es decir, continúa la exclusión de los conocimientos propios e incide en la pérdida progresiva de su identidad y el uso del mapunzugun, además, niega otra forma de conocer, comprender y dar sentido a la realidad social y cultural (RIVERA, 2010; SANTOS, 2009). Más aún, la dominación epistémica limita las perspectivas indígenas de concebir el conocimiento educativo en la institución escolar (KERR, 2014). Lo anterior refleja la violencia epistémica y curricular que subyace en la escuela, negando la racionalidad propia del conocimiento educativo del pueblo mapuche (MANSILLA; BECERRA; MERINO, 2015; QUILAQUEO; QUINTRIQUEO; PEÑA-CORTÉS, 2016).

\section{Alcances y limitaciones de la Educación Intercultural Bilingüe}

Los alcances de la EIB, según la literatura científica (GAUDET; LOSLIER, 2005; QUINTRIQUEO, 2009; HANNA MARCHIORO, 2011; DELGADILLO, 2015; PATRASCU; ALLAM, 2015), se relacionan con las siguientes dimensiones:

1) la sensibilidad, referida a construir actitudes de aceptación al otro respetando sus diferencias sociales, culturales, espirituales, cognitivas y lingüísticas en los actores del medio educativo y social (VILÁ, 2012);

2) comunicación intercultural, referida al aprendizaje de los códigos lingüísticos y culturales, para relacionarse adecuadamente con personas que pertenecen a sociedades y culturas diferentes (VILÁ, 2012);

3) relaciones interculturales, referida a la interacción, comunicación y diálogo que surge entre grupos sociales y culturales diversos que conviven en un espacio común, donde los saberes y conocimientos educativos propios, así como la lengua, se configuran en elementos constitutivos de la comunicación. Según Gómez y Hernández (2010) las relaciones interculturales que se viven en la educación escolar implican la confrontación de la cultura, saberes, conocimientos, valores y espiritualidad que portan los profesores, estudiantes indígenas y no indígenas como actores del medio educativo en que interactúan en el proceso educativo escolar; 4) pluralidad epistemológica, referida a la posibilidad de existencia de diferentes epistemes en un contexto donde coexisten diferentes grupos sociales y culturales que están en la base para la construcción de saberes y conocimientos para comprender la realidad social, cultural, natural y espiritual (ZAMBRANA, 2014); 
5) derecho a la educación propia que se configura en procesos de lucha y de resistencia de las comunidades indígenas para proteger su identidad y existencia, con la finalidad de revertir los procesos de sub-alternización, dominación, discriminación y colonización del ser, saber y poder que se ha impuesto a las comunidades indígenas (MOLINA; TABARES, 2014);

6) formación local en perspectiva global, para una vinculación de los sujetos con la enseñanza-aprendizaje en base a la epistemología tanto occidental como indígena para desenvolverse adecuadamente en contextos locales y globales (MOLINA; TABARES, 2014);

7) la relación familia-escuela-comunidad, en relación a la participación de los padres y miembros de las comunidades indígenas en las dinámicas escolares, para establecer entornos colaborativos que faciliten los procesos de enseñanzaaprendizaje, desde una mirada local (BASTIANI; BERMÚDEZ, 2015);

8) recursos didácticos y curriculares, respecto al material concreto, elementos tecnológicos e instrumentos que permitirían desarrollar el proceso de enseñanzaaprendizaje en contextos indígenas e interculturales de una manera contextualizada, en base a métodos de enseñanza apropiados y acordes al contexto educativo (CORTEZ, 2012).

En La Araucanía, los alcances logrados en la implementación de la educación intercultural bilingüe refieren a espacios de reconocimiento de la educación familiar mapuche en: 1) la incorporación del educador tradicional para apoyar la implementación de la EIB; 2) visibilizando el conocimiento mapuche en el medio escolar y académico; y 3) el desarrollo de investigaciones que están sistematizando saberes y conocimientos educativos mapuches pertinentes de incorporar al currículum escolar. No obstante, observamos que su implementación tanto a un nivel empírico y metodológico es incipiente, tal como observamos en los datos cualitativos que entrega un estudio encargado por el Ministerio de Educación (CHILE, 2012). En tanto, las limitaciones que se constatan, en el contexto de La Araucanía son: 1) discrepancia en las definiciones de identidad, cultura, interculturalidad, bilingüismo; 2) no existe una sistematización del trabajo realizado en el Programa de Educación Intercultural Bilingüe; 3) la EIB se plantea sólo para estudiantes de origen indígena; 4) se plantea a los pueblos indígenas como carentes, sin capacidad propositiva; 5) existe la idea de una asimilación de los indígenas a la sociedad nacional; 6) los planes de estudio integran la EIB como asignatura y no como modelo pedagógico; 7) en la implementación de la EIB no se consideran otros agentes educativos, como padres de familia y dirigentes de la comunidad; y 8) se ha excluido la EIB de la zona urbana (CHILE, 2012).

Las limitaciones señaladas reflejan que se sigue construyendo una representación negativa de lo mapuche en el desarrollo de la educación intercultural bilingüe. Por ejemplo, el reducirla sólo a contenidos lingüísticos genera una invisibilización de las representaciones sociales del pueblo mapuche, lo que descontextualiza epistemológicamente la EIB. Desde este argumento, es necesario no instrumentalizar el mapunzugun en función de significados de palabras, obviando las intersubjetividades que mediante el uso de la lengua permite entender y comprender el mundo. 
La EIB a nivel empírico no ha respondido a las demandas de las familias y comunidades mapuches, en relación a la incorporación de saberes y conocimientos educativos propios al sistema educativo escolar. Así, las demandas de las familias mapuches están subyugadas al poder que ejerce la escuela y la sociedad chilena sobre aquellos contenidos, métodos y finalidades educativas mapuches deseables de incorporar a la educación escolar. Constatamos así, que la gestión educativa aún está centralizada, excluye y margina a los estudiantes de origen mapuche de sus contextos sociales y culturales propios. Además, excluye a los estudiantes chilenos de origen no indígena, en su posibilidad de enriquecer su formación escolar desde el conocimiento educativo mapuche. Según Bastías (2014), la marginación de estudiantes mapuches y no mapuches es estructural, puesto que niega la interculturalidad y los derechos lingüísticos de los educandos mapuches que viven en sectores urbanos, por representar menos del 20\% de los estudiantes de cada establecimiento escolar. En este sentido, a la población indígena que constituye minoría sea en el contexto urbano como rural se le niega la posibilidad de aprender una lengua indígena en la escuela. De esta manera, se observa que existen contradicciones de la política pública que conllevan a una pérdida progresiva de las lenguas indígenas, de las culturas originarias y el aumento de prejuicios y estereotipos hacia los sujetos indígenas. En base a los argumentos anteriores, sostenemos que la EIB continúa bajo la hegemonía lingüística y cultural de la sociedad occidental, por sobre la sociedad mapuche (ÁLVAREZ-SANTULLANO; FORNO, 2008; QUILAQUEO; TORRES, 2013; QUINTRIQUE0 et al., 2015).

Desde la perspectiva descrita, creemos que al concebir la EIB sólo como una asignatura fragmentada del currículum escolar, se le niega a la lengua mapuche (mapunzugun) la posibilidad de transformarse en un objetivo pedagógico transversal en el sistema educativo escolar, sin embargo, esto promovería la incorporación de saberes y conocimientos educativos mapuches para la transformación de las prácticas educativas escolares (QUINTRIQUE0; TORRES, 2012). Sin embargo, la institución escolar históricamente, y aun en la actualidad, se ha encargado de transmitir solamente los valores e ideologías desde una epistemología occidental, cuya consecuencia ha sido la discriminación social, el racismo y la monoculturalidad de la escuela para el pueblo mapuche (BESALÚ, 2004). Esto refleja que la sociedad chilena no reconoce los saberes y conocimientos educativos que el pueblo mapuche puede aportar a la implementación de una educación intercultural, en articulación entre los saberes y conocimientos mapuches y occidentales.

La EIB a nivel metodológico, a pesar de abrir espacios para incorporar los saberes y conocimientos propios del pueblo mapuche a la institución escolar, no ha sido capaz de implementarse desde una postura crítica respecto del currículum escolar, donde se cuestione la visión estática que se tiene de los pueblos indígenas y sus culturas en los procesos de enseñanza-aprendizaje en la educación escolar (CHILE, 2011). Metodológicamente, la EIB al ser abordada desde una epistemología monocultural, termina homogeneizando o folclorizando los conocimientos y saberes del pueblo mapuche, perdiendo el sentido que estos tienen. De esta manera, no se logra que los estudiantes mapuches y no mapuches aprendan y desarrollen otras formas de pensar y comprender el mundo, puesto que la epistemología eurocéntrica occidental, en el medio escolar, no reconoce otra forma 
de ser, sentir y pensar. Por consiguiente, es necesario considerar los conocimientos y perspectivas de los pueblos indígenas sobre educación que aporten en la deconstrucción de las desigualdades sociales, el colonialismo, la opresión y la racionalidad monocultural subyacente en la escuela (BLANCHET; REILLYA, 2013).

En resumen, la influencia de la EIB, desde una epistemología occidental, ha sido estratégicamente implementada en la escuela para avanzar hacia un epistemicidio institucionalizado de los conocimientos indígenas (CORREA; SALDARIAGA, 2014). Es así como mediante la supuesta apertura a los conocimientos indígenas en la institución escolar, se promueve un epistemicidio encubierto de los saberes y conocimientos indígenas. Es decir, promoviendo la conformación de un nuevo sujeto indígena, construido en base a la subjetividad desde la lógica occidental. En este proceso, los sujetos indígenas pasan de ser sujetos de una epistemología propia, a ser los despojados de sus derechos, saberes y conocimientos, como también a ser sujetos discriminados por su singularidad (CORREA; SALDARIAGA, 2014). En consecuencia, la institucionalización del epistemicidio indígena mediante la educación escolar, legitima las desigualdades sociales, convirtiéndose en estrategias de control que permiten únicamente la incorporación de los saberes y conocimientos indígenas que no se contraponen con la lógica occidental.

En la perspectiva señalada, la educación escolar incorpora algunos bailes, canciones y traducción de palabras que no ponen en riesgo la estructura de poder y dominación hacia lo indígena (NASCIMENTO; QUADROS; FIALHO, 2016). Consideramos que la EIB desde esta ideología se desarrolla desde una mirada simplista, reduciendo las complejidades del conocimiento educativo indígena y de sus prácticas epistémicas. Por lo tanto, es imperativo romper con esta postura acrítica del sistema educativo escolar que ubica los conocimientos indígenas como contenidos homologables al conocimiento escolar (NASCIMENTO; QUADROS; FIALHO, 2016). Esto porque de esta forma se invisibilizan las especificidades propias de estos conocimientos que responden al espacio territorial en el cual han sido construidos y sus diferencias dependiendo de la identidad territorial. Desde este argumento, la historia enseñada es contada desde una epistemología occidental por parte del Estado estigmatizando las futuras generaciones indígenas (CORREA; SALDARIAGA, 2014). Es decir, como sujetos que no tienen arraigo en su propia identidad cultural, ni conocen la real participación de sus ancestros en la construcción de su historia local. Esto sería producto de la escolarización, ya que son los establecimientos escolares los que se han encargado de obstaculizar el reconocimiento de esas historias, ocultando cómo ocurrieron realmente los hechos históricos de la constitución del Estado-Nación. Así, desde la epistemología occidental se ha prescindido de cualquier otro tipo de conocimiento en la educación escolar. Y, en el caso que ocurra la más mínima visibilización de la historia local el Estado-nación se encarga de sancionar, inhabilitar, castigar, menospreciar y silenciar, mediante el currículum escolar único y las prácticas escolares monoculturales que emergen en la institución escolar. El fin último es ignorar e invisibilizar la episteme indígena atribuyéndole características de no ser un conocimiento válido, sino más bien retrasado que no responde a los cánones impuestos desde un paradigma científico. Remarcamos que la EIB al ser abordada desde una epistemología contextual, centrada en la población en general tales como sectores rurales, urbanos y en el ámbito social y 
educativo, permitiría a los estudiantes mapuches y no mapuches tener docentes que les enseñen en su lengua considerando aspectos sociales y culturales. Entonces, el desafío para los profesores es concebir la EIB como un compromiso personal, ético y político en el marco de la sociedad en general. Así, la EIB puede ser una herramienta que logre aportar el desarrollo indígena y no indígena con identidad, desde una epistemología contextual.

\section{Desafío de la EIB desde una epistemología contextual}

El desafío de la EIB es considerar una epistemología contextual en la definición de contenidos, métodos y finalidades educativas, para contrarrestar la monoculturalidad de la escuela y la hegemonía del conocimiento escolar. Este desafío supone aceptar la pluralidad de pensamiento y conocimiento como multiplicidad de singularidades (ZAMBRANA, 2014). Esto implica concebir la escuela como un espacio en el cual converge una multiplicidad de formas de pensar, conocer, concebir, imaginar y comprender la realidad educativa, en oposición al pensamiento colonial. Ello permitiría generar espacios de participación y colaboración de los actores del medio educativo y social, para la formación de todos los niños y adolescentes, independiente de su origen étnico, sociocultural y económico.

En consecuencia, para promover un giro decolonial hay que reapropiarse del pensamiento monocultural y universal en tanto crítica epistemológica (SANTOS, 2009). En el caso mapuche, el desafío epistemológico es abrirse a otras formas de construir conocimientos, de enseñar, aprender, dar sentido y significado a la realidad como resultado de la construcción social, incorporando la subjetividad y la espiritualidad que está en la base de la educación familiar, desde sus propios marcos epistémicos (QUILAQUE0; QUINTRIQUE0; PEÑA-CORTÉS, 2016). Esto permitiría transformar la monoculturalidad y cuestionar la hegemonía del conocimiento escolar en la escuela.

Entonces, mediante una epistemología contextual podríamos avanzar hacia una interculturalidad emancipadora, planteando un descentramiento respecto de la cultura dominante y concibiendo lo cultural desde una relación de saberes (SANTOS, 2009; QUILAQUEO; TORRES 2013; ZAMBRANA, 2014). Junto con ello, surge también la necesidad de repensar y cuestionarse la supremacía del conocimiento disciplinar occidental, basado en una lógica monocultural, instrumental y científica, lo que posibilitaría la incorporación de saberes y conocimientos indígenas a la educación escolar (PIZZI, 2005). Por ende, el pluralismo epistemológico nos lleva a una democracia de saberes y a la necesaria transformación de la estructura de la educación escolar, a una descolonización del conocimiento escolar y una valoración simétrica del conocimiento indígena en la educación escolar (ZAMBRANA, 2014). Es decir, reconocer que todos los conocimientos son dinámicos en la respuesta a los cambios y las presiones de la modernidad. Por lo cual no están congelados en el tiempo y el espacio, se basan continuamente en los desafíos cotidianos de la supervivencia humana y la forma de responder a las demandas sociales (DIE, 2011).

En resumen, la EIB fundada en una epistemología contextual debe considerar que las palabras no tendrán realidad en sí mismas, si son concebidas de forma externa a los sujetos implicados (QUILAQUEO; TORRES, 2013). Esta epistemología contextual pone el 
acento en los significados, valoraciones, saberes y conocimientos de los sujetos en relación a la memoria individual y social que poseen. Para ello, la institución escolar debe crear un contexto culturalmente sensible para el aprendizaje de todos los estudiantes, promoviendo una concepción de mundo transformadora en función de una sociedad más justa. Finalmente, consideramos que es posible incorporar epistemologías diversas en la educación escolar, que se contraponen entre sí, que son concurrentes y que podrían contribuir para estudiar y comprender la complejidad de pensar una EIB para contextos indígenas (JAMIOY, 1997; QUINTRIQUEO et al., 2015). Así, la epistemología contextual implica un proceso que se basa en la relación de saberes indígenas y occidentales, como respuesta al agotamiento progresivo de la racionalidad instrumental (FOLLARI, 2000; OLIVÉ, 2006; CASTELLANO; HINESTROZA, 2009; SANTOS, 2009; RIVERA, 2010; QUINTRIQUE0; TORRES, 2012). En efecto, constatamos que existen diferentes posibilidades para concebir la EIB desde una epistemología contextual que reoriente las concepciones en las cuales se han fundado los programas que han emergido de las políticas públicas, pero de cierta forma no han tenido efecto por estar profundamente compuestas desde una lógica positivista e instrumental.

\section{Discusión y conclusión}

El análisis de contenido de la literatura normativa y científica nos permite constatar que la EIB en Chile está instituida solamente en una epistemología de corte eurocéntrico occidental que no logra generar impacto en la formación de los educandos indígenas y no indígenas. Desde esa visión la EIB se convierte en una modalidad educativa con un alto componente cultural, cuya particularidad epistemológica ha pretendido generalizar y establecer la concepción del mundo a partir del marco cognitivo, valorativo y normativo, desde una visión única y hegemónica que se caracteriza por el uso exclusivo del español como lengua dominante. Esto limita la transformación de la institución escolar y más importante aún, el cambio de la sociedad en general. Lo anterior se refleja en el currículum real donde no se explicitan los conocimientos indígenas en el proceso de enseñanza-aprendizaje. Estos son elementos causantes de la inadecuada implementación y significación que se le ha dado a la EIB y lo que impide transformar y descolonizar los conocimientos que por años han formado a los ciudadanos chilenos.

Consideramos que una oportunidad de transformar la EIB pudiese ser avanzar en una epistemología contextual que considere la realidad local del entorno social en el cual se encuentra inserta la escuela, para avanzar en colaboración con los miembros de la familia y comunidad en la construcción social de una educación que responda a sus demandas e intereses. Esto permitiría a las personas mapuches y no mapuches conocer otras formas de construir conocimiento para comprender el mundo, sin sesgos y prejuicio. Asimismo, la EIB desde una epistemología contextual se configura en un imperativo ético, político y epistemológico para el Estado. De esta forma, a los estudiantes indígenas les permitiría revalorizar su sociedad y cultura, hasta aquí segregada y excluida por años en el sistema educativo escolar (GARCÍA, 2005; BASTÍAS, 2014).

En conclusión, una propuesta de EIB desde una epistemología contextual debe partir por los siguientes elementos: 1) generar espacios en la institución escolar para la 
co-construcción de materiales didácticos que sistematicen contenidos educativos propios, con el apoyo de profesores, familia y comunidad. Por ejemplo, construir un calendario de siembras, manuales didácticos que expliciten prácticas sociales y culturales propias de las comunidades, que describan sus significados, normas y pautas para la participación. Esto permitiría a los estudiantes mapuches y no mapuches, conocer los patrones culturales de dichas ceremonias, que podrían aplicar en situaciones de participación real; 2) incorporar métodos educativos mapuches propios a la implementación de la educación intercultural. Por ejemplo, explicitar en las dimensiones didáctica, pedagógica y curricular la importancia del aprender haciendo, observando y escuchando, para la formación de todos los estudiantes; 3) favorecer el trabajo colaborativo con los miembros de la comunidad, que tienen conocimientos sobre oficios, para articular el conocimiento educativo mapuche y el escolar en la sala de clases; y 4) promover procesos de enseñanza-aprendizaje que se realicen a partir de actividades de indagación tanto en la comunidad como en la familia y en laescuela. Esto permitiría la diversificación de los contenidos, además de romper con la estructura organizacional y del conocimiento disciplinario en la escuela, que no concibe espacios de aprendizaje fuera de la sala de clases. Asimismo, permitiría profundizar en los conocimientos y especificidades propias del territorio e historia local, en donde se encuentra inserta la escuela.

\section{Referencias}

ÁLVAREZ-SANTULLANO, Pilar; FORNO, Amilcar. La inserción de la lengua mapuche en el currículum de escuelas con educación intercultural: un problema más que metodológico. Alpha, Osorno, n. 26, p. 9-28, 2008.

BASTIANI, José; BERMÚDEZ, Flor. La participación de padres de familia en la Educación Indígena Intercultural Bilingüe (EIB) en la región Ch'ol de Chiapas. Revista Interamericana de Educación de Adultos, México, DC, v. 37, n. 1, p. 21-40, jun. 2015.

BASTÍAS, Rafael. Reflexiones en torno a la construcción de un currículum intercultural bilingüe desde un paradigma critico-liberador. Revista Isees, Santiago de Chile, n. 14, p. 79-92, 2014.

BESALÚ, Xavier. La formación inicial en interculturalidad. In: JORDÁN, José et al. (Org.). La formación del profesorado en educación intercultural. Madrid: MEC, 2004. Los libros de la catarata. Cuadernos de educación intercultural. p. 49-92.

BLANCHET, Natasha; REILLYA, Rosemary. Teachers' perspectives on environmental education in multicultural contexts: Towards culturally-responsive environmental education. Teaching and Teacher Education, Reino Unido, n. 36, p. 12-22, 2013.

CANDAU, María. Cotidiano escolar e práticas interculturais. Cadernos de Pesquisa, São Paulo, v. 46, n. 161, p. 802-820, 2016.

CASTELLANO, Ana; HINESTROZA, Jorge. Una perspectiva epistemológica para el estudio de las formaciones socioculturales. Revista de Ciencias Sociales, Valparaíso, v. 15, n. 4, P. 681-692, 2009.

CHILE. Ministerio de Educación. Programa de Educación Intercultural Bilingüe. Consulta a los pueblos indígenas informe de resultados: función docente del educador(a) tradicional para la implementación del sector de lengua indígena. Santiago de Chile: Ministerio de Educación, 2011. 
CHILE. Ministerio de Educación. Programa de Educación Intercultural Bilingüe. Educación para preservar nuestra diversidad cultural: desafíos de implementación del sector de lengua indígena en Chile. Santiago de Chile: Ministerio de Educación, 2012.

CORREA, Mario; SALDARIAGA, Dora. El epistemicidio indígena latinoamericano: algunas reflexiones desde el pensamiento crítico decolonial. Revista Ces Derecho, Medelin, v. 5, n. 2, p. 154-164, 2014.

CORTEZ, Fernando. Serie buenas prácticas sobre educación indígena. México, DC: Unicef, 2012.

DELGADILLO, Iris. Analyse du concept interculturel dans le discours pédagogique et l'enseignement des langues étrangéres en Colombie. Signes, Discours et Societes: semantiques des posibles argumentatifs et analyse linguistique du discours, Instambul, jan. 2015. Disponible en: <http: // www.revue-signes.info/ document.php?id=3887ISSN 1308-8378 >. Acceso en: 30 nov. 2015.

DIE, George. Integrating local cultural knowledge as formal and informal education for young African learners: a Ghanaian case study. Canadian and International Education, Ontário, v. 40, n. 1, p. 21-40, 2011.

FOLLARI, Roberto. Epistemología y sociedad: acerca del debate contemporáneo. Perfiles Educativos, México, DC, v. 22, n. 88, p. 1-124, 2000.

FORNET-BETANCOURT, Raúl. Filosofía e interculturalidad en América Latina, intento de introducción no filosófica. In: HEISE, María (Org.). Interculturalidad: creación de un concepto y desarrollo de una actitud. Lima: Inversiones Hathuey, 2001. p. 63-73.

GARCÍA, José. Educación intercultural análisis y propuestas. Revista de Educación, Madrid, n. 336, p. 89-109, 2005.

GAUDET, Edithe; LOSLIER, Sylvie. In thought and deed, committed to and intercultural Québec. Pedagogie Collegiale, Montreal, v. 19, n. 2, p. 5, 2005.

GÓMEZ, Jorge; HERNÁNDEZ, Juana. Relaciones interculturales, interculturalidad y multiculturalismo; teorías, conceptos, actores y referencias. Revista Cuicuilco, México, DC, v. 17, n. 48, p. 11-34, 2010.

HANNA MARCHIORO, Paola. Educação intercultural: limites e possibilidades no trabalho docente. In: CONGRESO NACIONAL DE EDUCACAO (EDUCERE), 1., 2011 [e] SEMINARIO INTERNACIONAL DE REPRESENTACOES SOCIAIS, SUBJETIVIDADE E EDUCACAO-SIRAAE, 10., 2011, Curitiba. Anais... Curitiba: Pontificia Universidad Católica do Paraná, 2011. p. 10828-10837.

HEVIA Ricardo; HIRMAS, Carolina. La política educacional intercultural bilingüe en Chile en el marco. Santiago de Chile: Cepal, 2005. Trabajo presentado en Seminario Internacional Pueblos Indígenas Afrodescendientes de América Latina y el caribe: relevancia y pertinencia de la información sociodemográfica para políticas y programas.

JAMIOY, José. Los saberes indígenas son patrimonio de la humanidad. Revista Nómada, Bogotá, v. 49, n. 7 , p. 64-72, 1997.

KERR, Jeannie. Western epistemic dominance and colonial structures: considerations for thought and practice in programs of teacher education. Decolonization, Toronto, v. 3, n. 2, p. 83-104, 2014.

LLANQUINAO, Gabriel. Revitalización lingüística del mapudungun: epistemología del hablante y metodologías propias. In: CONGRESO INTERNACIONAL DE LA SOCIEDAD CHILENA DE LINGÜÍSTICA, 20., 2009, Concepción. Concepción: Universidad Católica de la Santísima Concepción, 2009. p. 71-92. 
LÓPEZ, Enrique. Trece claves para entender la interculturalidad en la educación Latinoamericana. In: PRATS, Enrics (Org.). Multiculturalismo y educación para la equidad. Barcelona: Octaedro: OEI, 2007. p. 13-44.

LÓPEZ, Enrique; KÜPER, Wolfgang. La educación intercultural bilingüe en América Latina y el Caribe: balance y perspectivas. Revista Iberoamericana de Educación, Madrid, n. 20, p. 1-70, 2000.

MAMPAEY, Jelle; ZANONI, Patrizia. Reproducing monocultural education: ethnic majority staff's discursive constructions of monocultural school practices. British Journal of Sociology of Education, Reino Unido, v. 37 , n. 7, p. 928-946, 2015.

MANSILLA, Juan; BECERRA, Sandra; MERINO, María. Curriculum violence occidental knowledge hegemony in relation to indigenous knowledge. Procedia-Social and Behavioral Sciences, Reino Unido, v. 190, p. 434-439, 2015.

MOLINA, Víctor; TABARES, José. Educación propia: resistencia al modelo de homogeneización de los pueblos indígenas de Colombia. Polis, Santiago de Chile, n. 38, p. 1-20, 2014.

NASCIMENTO, Raimundo Ferreira do; QUADROS, Marión Teodósio de; FIALHO, Vania. Interculturalidade enquanto prática na educação escolar indígena. Revista Anthropológicas, Recife, v. 27, n. 1, p. 187-217, 2016.

OLIVÉ, León. Interculturality, Social Justice and Knowledge. Poligrafi, Eslovenia, v. 11, n. 41, p. 61-80, 2006.

OLIVÉ, León. Por una auténtica interculturalidad basada en el reconocimiento de la pluralidad epistemológica. In: OLIVÉ, León et al. (Orgs.) Pluralismo epistemológico. La Paz: Clacso: Muela del Diablo, 2009. p. 19-30.

OSORI0, Beatriz; APARECIDA, María. Elementos espirituais, simbólicos e afetivos na construção da escola mbyá guaranil. Educação e Pesquisa, São Paulo, v. 42, n. 4, p. 921-935, 2016.

PATRASCU, Ecaterina; ALLAM, Mohammad. Role of intercultural education in making a global world. Brasov: [s. n.], 2015. Trabajo presentado en International Conference RCI'15 Redefining Community in intercultural context, 21-23, mayo, 2015.

PIZZI, Jovino. El mundo de la vida: Husserl y Habermas. Santiago de Chile: Universidad Católica Silva Henríquez, 2005.

QUIJANO, Anibal. Colonialidad del poder modernidad racionalidad. Bogotá: Tercer Mundo, 1992.

QUIJANO, Anibal. Colonialidad del poder y clasificación social. Buenos Aires: Clacso, 2014.

QUILAQUEO, Daniel; QUITRIQUEO, Segundo; PEÑA-CORTÉS, Fernando. Interculturalidad en contexto de diversidad social y cultural: desafíos de la investigación educativa en contexto indígena. Temuco: Universidad Católica de Temuco, 2016.

QUILAQUEO, Daniel; QUINTRIQUEO, Segundo; TORRES, Héctor. Características epistémicas de los métodos educativos mapuches. Revista Electrónica de Investigación Educativa, México, DC, v. 18, n. 1, p. 153-165, 2016.

QUILAQUEO, Daniel; TORRES, Héctor. Multiculturalidad e interculturalidad: desafíos epistemológicos de la escolarización desarrollada en contextos indígenas. Alpha, Osorno, n. 37, p. 285-300, 2013. 
QUINTRIQUEO, Segundo. Posibilidades y limitaciones de la educación intercultural en contexto mapuche. Cuhso, Temuco, v. 17, n. 1, p. 23-35, 2009.

QUINTRIQUEO, Segundo; MUÑOZ, Gerardo. Demandes educatives éducationelles sociohistoriques et éducation interculturelle en contexte mapuche. In: SALAS, Ricardo; LE BONNIEC, Fabiám (Org.). Les mapuche á la mode: modes d'existence et de résistance au Chili, en Argentine et au-delá. Paris: L'Harmattan, 2015. p. 95-122.

QUINTRIQUEO Segundo; TORRES Héctor. Construcción de conocimiento mapuche y su relación con el conocimiento escolar. Estudios Pedagógicos, Valdivia, v. 39 n. 1, p. 199-216, 2012.

QUINTRIQUEO, Segundo et al. Competencia comunicativa intercultural: formación de profesores en el contexto poscolonial chileno. Alpha, Osorno, n. 45, p. 235-254, 2017.

QUINTRIQUEO, Segundo et al. Conocimientos culturales como contenidos de la educación familiar mapuche. Alpha, Osorno, n. 40, p. 131-146, 2015.

RIEDEMANN, Andrea. La educación intercultural bilingüe en Chile: ¿ampliación de oportunidades para alumnos indígenas? Revista Indiana, Berlín, n. 25, p. 169-193, 2008.

RIVERA, Silvia. Ch'ixinakax utxiwa: una reflexión sobre prácticas y discursos descolonizadores. Buenos Aires: Tinta Limón, 2010.

SALAÜN, Marie. Décoloniser l'école?: Hawai'i, Nouvelle-Calédonie: expériences contemporaines. Rennes: PUR, 2013.

SANTOS, Boaventura. Descolonizar el saber, reinventar el poder. Montevideo: Trilce-Extensión, 2009.

SARANGAPANI, Patricia. Savoir, curricula et méthodes pédagogiques: le cas de l'Inde. Revue Internationale d’Éducation de Sèvres, Sèvres, 2014. Colloque: L'éducation en Asie en 2014: Quels enjeux mondiaux? Disponible en: <https://ries.revues.org/3851?lang=es>. Acceso en: 05 jun. 2014.

SAYAGO, Sebastián. El análisis del discurso como técnica de investigación cualitativa y cuantitativa en las ciencias sociales. Cinta Moebio, Santiago de Chile, n. 49, p. 1-10, 2014.

SERRANO, Sol; PONCE DE LEÓN, Macarena; RENGIFO, Francisca. Historia de la educación en Chile: aprender a leer y escribir (1810-2010). Tomo I. Santiago de Chile: Taurus, 2012.

SILVA-PEÑA, llish et al. Sentido de la escuela para niños y niñas mapuche en una zona rural. Polis, Santiago de Chile, v. 12, n. 34, p. 243-258, 2013.

TUBINO, Fidel. El trasfondo epistémico de los conflictos interculturales. Contextualizaciones Latinoamericanas, Guadalajara, v. 2, n. 11, p.1-6, 2014.

TUBINO, Fidel. Intercultural practices in Latin American nation states. Journal of intercultural studies, Reino Unido, v. 34, n. 5, p. 604-619, 2013. 
TURRA, Omar; FERRADA, Donatila; VILLENA, Alicia. La especificidad del contexto indígena como requerimiento para la formación inicial del profesorado. Estudios Pedagógicos, Valdivia, v. 39, n. 2, p. 329-339, 2013.

VAN DIJK, Teun. Prejuicio en el discurso: análisis del prejuicio étnico en la cognición y en la conversación. Sevilla: Arcibel, 2010.

VILÀ, Ruth. Los aspectos no verbales en la comunicación intercultural. Ra Ximhai, México, DC, v. 8, n. 2, p. 223-239, 2012.

VIVEROS-MÁRQUEZ, José; MORENO-OLIVOS, Tiburcio. El enfoque intercultural bilingüe y su impacto en la calidad de la educación indígena: estudio de caso. Ra Ximhai, México, DC, v. 10, n. 3, p. 55-73, 2014.

WALSH, Catherine. "Other" knowledges, "Other" critiques: reflections on the politics and practices of philosophy and decoloniality in the "Other" America. Transmodernity, California, n. 1, p. 2154-1361, 2012 a.

WALSH, Catherine. The plurinational and intercultural state: decolonization and state re-founding in Ecuador. Bulletin of the Peoples' Friendship University of Russia, Cincinnati, n. 1, p. 65-84, 2012b.

WILLIAMSON, Guillermo. Institucionalización de la educación intercultural bilingüe en Chile: notas y observaciones críticas. Perfiles Educativos, México, DC, n. 138, p. 126-147, 2012.

ZAMBRANA, Amilcar (Ed.). Pluralismo epistemológico: reflexiones sobre la educación superior en el estado plurinacional de Bolivia. Cochabamba: Funproeib, 2014.

Recibido en: 09.03.2018

Aprobado en: 16.05.2018

Katerin Elizabeth Arias Ortega es doctora en Educación. Académica del Departamento de Psicología de la Facultad de Ciencias de la Salud de la Universidad Católica de Temuco (UCT). Investigadora Joven del Centro de Investigación en Contexto Indígena e Intercultural (CIECII) de la UCT.

Daniel René Quilaqueo Rapimán es doctor en Sociología. Director del Programa de Doctorado en Educación en Consorcio de la Universidad Católica de Temuco (UCT). Director del Centro de Investigación en Contexto Indígena e Intercultural (CIECII) del Núcleo de Estudios Interétnicos e Interculturales (NEII) de la UCT e investigador asociado del Centre Interuniversitaire d'Études et de Recherches Autochtones (CIÉRA) de I'Université Laval, Québec, Canadá.

Segundo Enrique Quintriqueo Millán es doctor en Educación, Universidad de Extremadura, España. Magíster en Educación, Universidad de Québec en Abitibi-Témiscamingue. Investigador titular del CIECII e investigador asociado del Centre Interuniversitaire d'Études et de Recherches Autochtones (CIÉRA) de I'Université Laval, Québec, Canadá. 